\title{
Von der Arbeitsgruppe AGQ-FMH zum Programm «Qualität FMH»
}

Ludwig T. Heuss, Georg von Below

Die Arbeitsgruppe Qualität AGQ-FMH (gegründet auf Antrag der Ärztekammer vom 19. Mai 2005 [1]) traf sich im September zu ihrer zweiten Sitzung. Ihre Mitglieder hatten bereits vorher zusammen das «Leitbild ärztliche Qualität» erarbeitet [2]. Darauf basiert der Aufbau des zukünftigen Programms «Qualität FMH». Dieser Artikel soll einen Einblick in die laufende Arbeit ermöglichen.

\section{Zielsetzungen}

Die bei der Gründung der AGQ-FMH definierten Aufträge wurden zu den folgenden Zielsetzungen spezifiziert:

\section{Leitbild weiterentwickeln}

- Definitionshoheit im Bereich Qualität erhalten;

- Impulse zur Begriffklärung und zur Qualitätsentwicklung innerhalb der Ärzteschaft erarbeiten und mit den Betroffenen umsetzen;

- Grundlagen, Argumente und Vorgehen zur systematischen Lösungserarbeitung entwikkeln.

\section{Umsetzung des Leitbildes fördern}

- Grundlagen und Argumente für das Zusammenspiel von Regulation, nachgewiesenen Fähigkeiten und Resultaten erarbeiten;

- Systemfunktionalität (gesamte Versorgungskette) als ein zentrales Ziel etablieren.

Konzepte für eine Diskussionsplattform Qualität für die Ärzteschaft sowie für die Ausbildung der Qualitätsbeauftragten und der Qualitätsaudits erarbeiten

- Medizinische Qualitätsentwicklungsprogramme evaluieren;

Korrespondenz:

Verbindung der Schweizer Ärztinnen und Ärzte FMH

Abteilung Daten, Demographie

und Qualität DDQ

Elfenstrasse 18

CH-3000 Bern 15

E-Mail: fmhqualitaet@hin.ch
- die FMH bei Stellungnahmen, politischen Vorstössen usw. unterstützen;

- Weiterbildungsveranstaltungen für Q-Verantwortliche planen und durchführen;

- Best-practice-Beispiele aufsuchen, überprüfen und bereitstellen.

Auf der Grundlage des Qualitätsverständnisses der Schweizer Ärzteschaft mit den Ansprechpartnern in Verhandlungen treten

- Relevante Player einbinden mit dem Ziel einer gemeinsamen Problemwahrnehmung und eines Konsenses bezüglich Rollenverteilung und Vorgehensweise bei der Lösungsfindung;

- Resonanzgruppe für die FMH bei Qualitätsfragen sein;

- mit Partnern eine gemeinsame inhaltliche Qualitätsplattform erarbeiten.

\section{Aufbau und Integration des Programms in die FMH}

Die Aufbauphase der AGQ-FMH ist bis Mitte 2006 vorgesehen. Danach laufen die Aktivitäten im Sinne eines Programms «Qualität FMH» weiter. Im Hinblick auf die angestrebte langfristige Perspektive ist die Klärung der Strukturen und Verantwortlichkeiten in der Anfangsphase unabdingbar. Abbildung 1 zeigt den Aufbau in Steuerungsgruppe, Programmleitung und Expertenpool sowie die Einbettung in die FMH.

\section{Wer macht was?}

Die Steuerungsgruppe hat unter der Leitung von ZV-Mitglied PD Dr. med. Ludwig T. Heuss unter anderem die Aufgaben, Inhalte zu definieren und Projektaktivitäten zu priorisieren. Wichtig ist es, dass die verschiedenen Projektvorschläge in einem Gesamtprogramm zusammengefasst sind, das die strategische Ausrichtung des Programms widerspiegelt. Weiter wird die Steuerungsgruppe die Ziele des Programms definieren, diese überprüfen und bei Bedarf Korrekturen ver- 
Abbildung 1

Organigramm des FMH-Programms «Qualität».

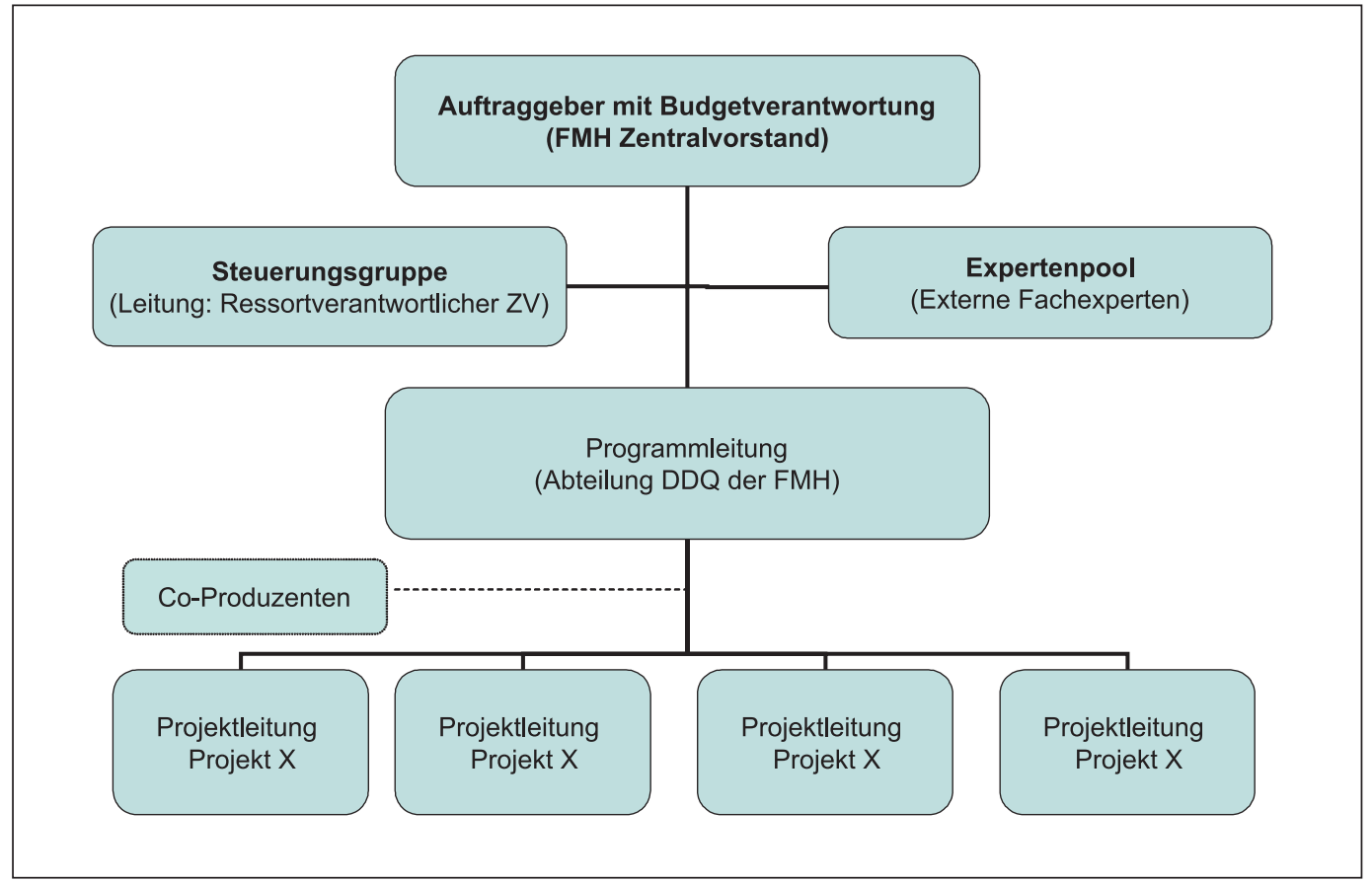

anlassen, Stellungnahmen zu Fachinhalten und neuen Entwicklungen abgeben sowie das Anspruchsgruppenmanagement sichern. Einzelne Mitglieder der Steuerungsgruppe können sich ausserdem in Projekten mit einbringen. Die Programmleitung übernimmt die Abteilung Daten, Demographie und Qualität (DDQ) der FMH. Sie arbeitet die projektbezogenen Umsetzungsvorschläge aus und ist für die programmbezogene Kommunikation verantwortlich.

Die kommende Zeit wird einerseits von der Gestaltung des Gesamtprogramms sowie der Definition der Prozesse in und rund um die AGQFMH bzw. das Programm «Qualität FMH» ge- prägt sein. Andererseits sind bereits die ersten Projekte in Angriff zu nehmen. Zweifellos handelt es sich für alle Beteiligten um eine spannende Phase!

\section{Literatur}

1 Müller Imboden A. Protokoll der ordentlichen Ärztekammersitzung (19. Mai 2005). Schweiz Ärztezeitung 2005;86(27):1625-31.

2 Peltenburg M, Kernen H, Schneider P, von Below GC, Waldis G, et al. Qualität - ein Zusammenspiel aller Kräfte im ärztlichen Umfeld. Schweiz Ärztezeitung 2005;86(18):1055-8. 